\title{
BMJ Open Existing predictive methods applied to gait analysis of patients with diabetes: study protocol for a systematic review
}

\author{
Patrícia Mayara Moura da Silva (1) , , ${ }^{1,2}$ Ana Beatriz Oliveira Bezerra, ${ }^{1}$ \\ Luanna Barbara Araújo Farias, ${ }^{1}$ Tatiana Souza Ribeiro, ${ }^{1}$ Edgard Morya, ${ }^{2}$ \\ Fabrícia Azevêdo da Costa Cavalcanti ${ }^{1}$
}

To cite: Moura da Silva PM, Oliveira Bezerra AB, Araújo Farias LB, et al. Existing predictive methods applied to gait analysis of patients with diabetes: study protocol for a systematic review. BMJ Open 2022;12:e051981. doi:10.1136/ bmjopen-2021-051981

- Prepublication history and additional supplemental material for this paper are available online. To view these files, please visit the journal online (http://dx.doi.org/10.1136/ bmjopen-2021-051981)

Received 01 April 2021 Accepted 18 January 2022

Check for updates

(C) Author(s) (or their employer(s)) 2022. Re-use permitted under CC BY-NC. No commercial re-use. See rights and permissions. Published by BMJ.

${ }^{1}$ Physical Therapy Department, Federal University of Rio Grande do Norte, Natal, Brazil

${ }^{2}$ Edmond and Lily Safra International Institute of Neurosciences, Santos Dumont Institute, Macaíba, Brazil

Correspondence to

Patrícia Mayara Moura da Silva; patriciamayarams@gmail.com

\section{ABSTRACT}

Introduction Type 2 diabetes can lead to gait abnormalities, including a longer stance phase, shorter steps and improper foot pressure distribution. Quantitative data from objective methods for evaluating gait patterns are accurate and cost-effective. In addition, it can also help predictive methods to forecast complications and develop early strategies to guide treatments. To date, no research has systematically summarised the predictive methods used to assess type 2 diabetic gait. Therefore, this protocol aims to identify which predictive methods have been employed to assess the diabetic gait.

Methods and analysis This protocol will follow the Preferred Reporting Items for Systematic Review and Meta-Analysis Protocol (PRISMA-P) statement. Electronic searches of articles from inception to January 2022 will be performed, from May 2021 to 31 January 2022, in the Web of Science, MEDLINE, Embase, IEEE Xplore Digital Library, Scopus, CINAHL, Google Scholar, APA Psyclnfo, the Cochrane Library and in references of key articles and grey literature without language restrictions. We will include studies that examined the development and/or validation of predictive methods to assess type 2 diabetic gait in adults aged $>18$ years without amputations, use of assistive devices, ulcers or neuropathic pain. Two independent reviewers will screen the included studies and extract the data using a customised charting form. A third reviewer will resolve any disagreements. A narrative synthesis will be performed for the included studies. Risk of bias and quality of evidence will be assessed using the Prediction Model Risk of Bias Assessment Tool and the Transparent Reporting of a multivariable prediction model for Individual Prognosis or Diagnosis.

Ethics and dissemination Ethical approval is not required because only available secondary published data will be analysed. The findings will be disseminated through peer-reviewed journals and/or presentations at relevant conferences and other media platforms.

PROSPERO registration number CDR42020199495.

\section{INTRODUCTION}

Diabetes mellitus is a worldwide health concern, with a prevalence of $8.8 \%$ in $2017 .{ }^{1}$ With type 2 diabetes mellitus being the most common, ${ }^{2}$ this condition is related to a dysfunction either in the pancreatic $\beta$-cells' ability to secrete

\section{Strengths and limitations of this study}

- This study will be the first systematic review to comprehensively analyse the existing predictive methods for gait analysis in patients with type 2 diabetes.

- This systematic review will focus on the predictive method's performance (surrogate outcomes) rather than on patient-reported outcome measures.

- A broad search strategy and robust quality assessment criteria (Transparent Reporting of a multivariable prediction model for Individual Prognosis or Diagnosis) will be used to appraise and examine the existing literature.

- Two independent reviewers will be responsible for conducting the study selection, data extraction and quality assessment.

- A limitation could be the potential lack of studies that meet the established inclusion criteria.

insulin, insulin resistance in target organs or a combination of both, resulting in hyperglycaemia. ${ }^{13}$ Patients can also present with blood vessel degeneration ${ }^{45}$ that can evolve into neuropathy and damage sensory and motor nerve fibres. ${ }^{4}$ Diabetes also alters physical function and mobility. ${ }^{6}$ Both can lead to motor abnormalities such as longer stance time (ie, greater support base) and shorter steps, which may exhibit as slower gait speeds and increased cadence. ${ }^{78}$ In addition, changes in the sensibility of the plantar surface of the foot can worsen plantar pressure distribution, balance and gait. ${ }^{57}$

Boosting insight into diabetic gait pattern alterations can be important for preventing complications caused by diabetes and developing strategies to guide treatments. ${ }^{6}{ }^{9}$ In clinical practice, while there is a high prevalence of observational methods, ${ }^{1011}$ this may be unreliable in assessing and diagnosing gait patterns. Observational methods are subjective and can generate inaccuracies during the assessment and diagnosis of the patient's movements due to different interpretations 
between examiners. In addition, these differences can impair decision-making to address a specific treatment. ${ }^{12-14}$ On the contrary, objective methods are reliable, accurate, quicker and cost-effective owing to quantitative metric results. ${ }^{8}$

Objective gait analysis methods require data collected from patients wearing sensors or performing gait in specific devices, such as inertial measurement units (IMUs), electromyography (EMG), optoelectronic systems or force platforms. ${ }^{10}$ Data from quantitative gait measures can be analysed using various methods. One of these is the use of predictive analytics that combine the collected data and estimate probabilities that can assist clinicians and potentially influence their decision to manage treatments to restore gait. ${ }^{15-18}$ Predictive methods are mathematical equations (from statistics or machine learning (ML) approaches) that can combine information from a set of data, resulting in a response forecasting the probability of a particular outcome. ${ }^{19} 20$

Emerging predictive methods include ML algorithms. ML can be used for automatic gait recognition to predict possible complications such as the risk of falls and pressure ulcers. ${ }^{21}$ Newer methods have opened new perspectives for the early diagnosis of gait disorders. This is essential in preventing potential future complications and to draw on personalised gait training ${ }^{15}$ by quantifying the treatment progress and follow-ups. ${ }^{22}$

To our knowledge, no research has systematically summarised predictive algorithms used to assess gait in patients with type 2 diabetes. Based on this, we raise an important question about the existence of predictive methods used to evaluate the gait of patients with type 2 diabetes. Therefore, the purpose of this study is to conduct a systematic review of the literature to summarise the evidence regarding existing predictive methods used in the gait patterns of patients with diabetes. In addition, we intend to describe the characteristics of the studies identified among the variety of gait data collected regarding which input features are the most commonly used to implement a predictive method.

\section{METHODS AND ANALYSES \\ Study design}

This systematic review protocol was prepared using the Preferred Reporting Items for

Systematic Reviews and Meta-Analysis Protocols (PRISMA-P). ${ }^{23}$

\section{Study registration}

This protocol was registered with the International Prospective Register of Systematic

Reviews (PROSPERO). Available from: https://www. crd.york.ac.uk/prospero/display_record.php?ID= CRD42020199495

\section{Eligibility criteria}

Types of study

Articles will be eligible for review when they describe the development and/or validation of a predictive method to assess gait in human type 2 diabetes. Furthermore, all published and unpublished studies (eg, dissertations and theses), conference proceedings that deal with diabetic gait analysis, independent of the parameters measured, will be included if developed and/or validated as a predictive method. There will be no geographical or language restrictions. Wherever necessary, relevant articles will be arranged for translation.

\section{Participants}

We will include clinical data from adult participants $(>18$ years old) who had type 2 diabetes diagnosed at any disease stage without lower limb amputations or the use of gait assistive devices. In addition, data with participants with ulcers or neuropathic pain (that could have interfered in the gait execution) will be excluded. There will be no restrictions on gender or race.

\section{Outcome measures}

The primary outcome will comprise all predictive methods (eg, ML models) applied to analyse gait in patients with type 2 diabetes. The secondary outcome will include gait data input features (eg, spatiotemporal, angular gait parameters, EMG data, force data and plantar pressure data) most commonly used to implement a predictive model.

\section{Search strategy for the identification of relevant studies}

The search strategy will be guided by the PRISMA extension for searching (PRISMA-S). ${ }^{24}$ The following electronic databases will be searched: Web of Science (Clarivate Analytics), MEDLINE (PubMed), Embase (Elsevier), IEEE Xplore Digital Library (IEEE), Scopus (Elsevier), CINAHL (EBSCOhost), Google Scholar (Google), APA PsycInfo (APA PsycNet) and the Cochrane Library (Wiley) from May 2021 to 31 January 2022. The time range of the published studies was from inception to January 2022. We will manually search the reference list of the studies included in the review. Grey literature involving published and unpublished studies (eg, dissertations and theses) and conference proceedings will also be searched without language restrictions, but this must be limited to human participants.

The articles will be searched using a combination of free keywords and the terminology registered in the Medical Subject Headings of the US National Library of Medicine. The terms that will be used are related to diabetes (eg, 'Type 2 Diabetes', 'Diabetes, Type 2', 'Diabetes Mellitus, Type 2'), gait (eg, 'Gait', 'Gait Analysis', 'Kinematic', 'Kinetic', 'Range of Motion') and prediction (eg, 'Artificial Intelligence', 'Machine Learning', 'Statisticallearning', 'Predictive Value of Tests', 'Support Vector Machine', 'Neural Networks, Computer'). The search strategy was pilot tested and finalised in MEDLINE 


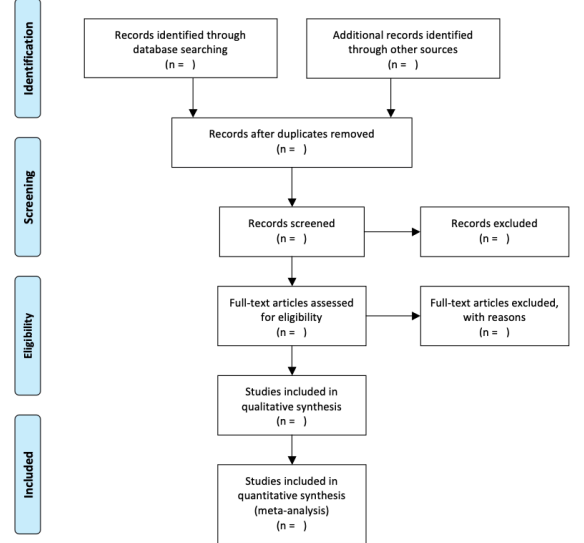

Figure 1 Preferred Reporting Items for Systematic Reviews and Meta-Analysis flow diagram for the identification, screening and eligibility of included articles.

(PubMed) before being translated for use in other databases. Details of the search strategies are provided in online supplemental appendix 1 .

\section{Screening of the studies}

Based on the previously described inclusion criteria, two independent reviewers (PMMdS, ABOB) will screen titles and abstracts identified during electronic and manual searches to determine its eligibility. Study record information, including title and abstract from the searched online database, will be imported into the Rayyan systematic review software. ${ }^{25}$ This platform will guide the reviewers in conducting the literature review process through its ability to explore and filter searched studies. Duplicate studies will be removed. If the title or abstract does not provide enough information for inclusion, the full text will be obtained for a full review. The same two reviewers (PMMdS, ABOB) will independently screen the full-text articles to identify studies for inclusion and record the reasons for exclusion for ineligible studies. Any disagreements that arise will be resolved initially by a discussion between the two reviewers, or, if necessary, with assistance from a third reviewer (FAdCC).

All reasons for the exclusion of ineligible studies will be recorded. The results of the screening process will be provided in detail using the PRISMA information flow chart (figure 1).

\section{Data extraction}

A data extraction form was developed through a discussion among all authors and adapted from the critical appraisal and data extraction for systematic reviews of prediction modelling studies (CHARMS) checklist. ${ }^{26}$ The included studies will go forward to the data extraction and quality assessment stages of the review. Two independent reviewers (PMMdS, $\mathrm{ABOB}$ ) will extract the outcome data from the included studies. If necessary, disagreements in data extraction will be discussed between the two reviewers and judged by a third reviewer (FAdCC).

The data collection form will aim to extract the key features of the review. Hence, we will divide the items within the data collection form into four blocks: (1) study information including publication year, author information, funding or sponsorship information, type of study, journal name and population, intervention, control and outcome (PICO elements); (2) database information including name, sample size, host organisation and sponsorship; (3) patient demographic information including gender, age, race and disease severity and (4) predictive methodological information including the type of gait assessment, comparisons with gold standard devices, type of predictive algorithm used (including its statistical or ML model name), format of input feature, optimisation algorithm, objective function, feature extraction methods, type of extraction feature and computational efficiency, and cost. Table 1 presents an example of the data extraction form. These data will be presented in the 'Characteristics of included studies' table.

Missing data may include missing outcomes, missing summary data or missing individual results. The authors will consider the reasons for the missing data. Where possible, we will contact the original investigators to obtain any missing data. However, in the case of contact difficulty, we will present the findings according to the statistical information available in each review, and this will be clearly stated in the final overview.

\section{Quality of evidence}

The quality of the predictive model used on the eligible studies will be assessed based on Transparent Reporting of a multivariable prediction model for Individual Prognosis or Diagnosis (TRIPOD) checklist. ${ }^{27}$ The TRIPOD Statement is a checklist of 22 items for the appropriate reporting of studies developing or validating multivariable prediction models. ${ }^{19}$ Each item will be scored as 0 , 1 and 2, ranked as 'no report', 'inadequate report' and 'adequate report', respectively.

\section{Risk of bias}

The preselected articles will be evaluated and scored for methodological quality using the Prediction Model Risk of Bias Assessment Tool (PROBAST) ${ }^{20}$ by two independent reviewers (PMMdS, ABOB). In cases of opinion divergence, a third reviewer (FAdCC) will decide the score. The questionnaire consists of 20 items with four domains (participants, predictors, outcome and analysis). Based on the questionnaire ratings, the risk of bias for each domain will be ranked as 'low risk', 'high risk' or 'too unclear for judgement'. PROBAST will be used to categorise the included studies regarding their methodological quality, but these studies will not be excluded based solely on this evaluation.

\section{Strategy for data synthesis}

A narrative synthesis will be conducted with the information presented in the text and table to summarise and explain the characteristics and findings of the included studies. Data will be summarised using descriptive statistics and visual plots. Categorical data about the reporting, 
Table 1 Example of the data extraction form for all included studies

\begin{tabular}{|c|c|}
\hline \multicolumn{2}{|l|}{ Study information } \\
\hline Study year & Year of the study publication \\
\hline Author information & Last name of the author, whether clinical practitioners participated in the study \\
\hline Type of study & Source of data (eg, cohort, case-control, randomised trial participants or registry data) \\
\hline Journal name & Journal name \\
\hline PICO elements & PICO elements in summary \\
\hline \multicolumn{2}{|c|}{ Database information } \\
\hline Database name & Name of the database used for modelling \\
\hline Host organisation & Name of the hosting organisation of the database \\
\hline Sponsorship & The funding or sponsorship information \\
\hline Sample size & Sample size used for building the model \\
\hline Source or data & $\begin{array}{l}\text { From which source the database was used (eg, electronic health records, clinical registry, administrative data, cohort } \\
\text { study, clinical trial) }\end{array}$ \\
\hline \multicolumn{2}{|c|}{ Patient demographic information } \\
\hline Gender & Sex of adults (male, female, alternative gender) \\
\hline Age & Age and/or year of birth \\
\hline Country & Country or countries in which study was based \\
\hline Diabetes severity & Disease severity \\
\hline
\end{tabular}

\begin{tabular}{|c|c|}
\hline \multicolumn{2}{|c|}{ Predictive methodological information } \\
\hline Predictors & Timing of predictor measurement (eg, at patient presentation, at diagnosis, at treatment initiation) \\
\hline Number of features & Number of features for building the model \\
\hline Type of extracted feature & Which features the algorithm uses (eg, pressure, gait velocity, cadence, step width, pixel feature, action unit) \\
\hline $\begin{array}{l}\text { Tool used for gait } \\
\text { assessment }\end{array}$ & Quantitative tool used to assess gait kinetic or kinematic (eg, IMU, force platform, optoelectronic, EMG) \\
\hline Predictive method used & Type of predictive method used to assess gait (eg, which machine learning techniques were used) \\
\hline Model name & $\begin{array}{l}\text { The name of the predictive model used. The underlying mathematical model used (eg, linear regression, support vector } \\
\text { machine) }\end{array}$ \\
\hline Missing data & $\begin{array}{l}\text { Number of participants with missing data for each predictor and the process handled with missing data (eg, complete- } \\
\text { case analysis, imputation or other methods) }\end{array}$ \\
\hline Model evaluation & $\begin{array}{l}\text { Method used for testing model performance: development dataset only (random split of data, resampling methods, } \\
\text { eg, bootstrap or cross-validation) or separate external validation (eg, temporal, geographical, different setting, different } \\
\text { investigators) }\end{array}$ \\
\hline $\begin{array}{l}\text { Computational efficiency } \\
\text { and cost }\end{array}$ & $\begin{array}{l}\text { Computational efficiency (speed, cloud space, etc) and cost related to the algorithm (eg, require GPU resources, large } \\
\text { cluster) }\end{array}$ \\
\hline
\end{tabular}

EMG, electromyography; GPU, graphics processing unit; IMU, inertial measurement unit; PICO, population, intervention (exposure), control, outcome.

methodological conduct and risks of bias will be described using numbers and percentages. The distribution of continuous data, such as sample sizes and the number of features, will be described using measures of central tendency such as mean and SD for normally distributed data and median and percentiles (25th and 75th) for non-normally distributed data.

The risk of bias assessment will be summarised and graphically presented for each PROBAST domain and the overall risk of bias judgement. The results will be stratified by prevalent predictive techniques and study design (development with internal validation and/or external validation). The quality of evidence based on TRIPOD will also be summarised and graphically presented for each included study and its respective score rank.

\section{Analyses of subgroups or subsets}

We plan to conduct subgroup analyses using predictive model types (eg, regression models vs classification models, neural networks vs traditional ML models) and gait input parameters (eg, kinematic vs kinetic data features, IMUs vs EMG data features). In addition, we 
plan to classify participants according to their anthropometric subgroup (eg, age, body index mass, height, weight, gait measurements and diabetes vitals). More exploratory subgroup analyses will be decided during the data extraction and analysis process.

\section{ETHICS AND DISSEMINATION}

To the best of our knowledge, this systematic review is the first that will synthesise existing evidence regarding the types of predictive methods used to assess gait in patients with type 2 diabetes. Predictive methods are increasingly being appraised and recommended for formal risk assessment in treatment decision-making and clinical guidelines. The proposed systematic review may inform future research and clinicians. For instance, it may help researchers in designing customisable prediction tools to be used in diabetic care, and thus allow physiotherapists to better conduct rehabilitative gait treatments in the patients with type 2 diabetes.

Because we will be using secondary data sources, ethical approval is not required for this systematic review study. Our findings will be disseminated through peer-reviewed publications, presentations at conferences and clinical and patient networks.

Acknowledgements The authors thank CAPES Foundation, an agency under the Ministry of Education (MEC) of Brazil for their assistance during the research.

Contributors PMMdS, ABOB, LBAF, TSR, EM and FAdCC wrote the main manuscript text. PMMdS prepared the figure and table. All authors reviewed the manuscript. All authors read and approved the final manuscript.

Funding This study was financed in part by the Coordenação de Aperfeiçoamento de Pessoal de Nível Superior - Brasil (CAPES) - Finance Code 001.

Competing interests None declared.

Patient consent for publication Not required.

Ethics approval This study does not involve human participants.

Provenance and peer review Not commissioned; externally peer reviewed.

Data availability statement No data are available. The study is a protocol for a systematic review. Thus, no data are available.

Supplemental material This content has been supplied by the author(s). It has not been vetted by BMJ Publishing Group Limited (BMJ) and may not have been peer-reviewed. Any opinions or recommendations discussed are solely those of the author(s) and are not endorsed by BMJ. BMJ disclaims all liability and responsibility arising from any reliance placed on the content. Where the content includes any translated material, BMJ does not warrant the accuracy and reliability of the translations (including but not limited to local regulations, clinical guidelines, terminology, drug names and drug dosages), and is not responsible for any error and/or omissions arising from translation and adaptation or otherwise.

Open access This is an open access article distributed in accordance with the Creative Commons Attribution Non Commercial (CC BY-NC 4.0) license, which permits others to distribute, remix, adapt, build upon this work non-commercially, and license their derivative works on different terms, provided the original work is properly cited, appropriate credit is given, any changes made indicated, and the use is non-commercial. See: http://creativecommons.org/licenses/by-nc/4.0/.

ORCID iD

Patrícia Mayara Moura da Silva http://orcid.org/0000-0002-4055-3289

\section{REFERENCES}

1 Standl E, Khunti K, Hansen TB, et al. The global epidemics of diabetes in the 21st century: current situation and perspectives. Eur J Prev Cardiol 2019;26:7-14.
2 Skyler JS, Bakris GL, Bonifacio E, et al. Differentiation of diabetes by pathophysiology, natural history, and prognosis. Diabetes 2017;66:241-55.

3 Chatterjee S, Khunti K, Davies MJ. Type 2 diabetes. Lancet 2017;389:2239-51.

4 Sawacha Z, Cristoferi G, Guarneri G, et al. Characterizing multisegment foot kinematics during gait in diabetic foot patients. $J$ Neuroeng Rehabil 2009;6:37.

5 Huang C-K, Shivaswamy V, Thaisetthawatkul P, et al. An altered spatiotemporal gait adjustment during a virtual obstacle crossing task in patients with diabetic peripheral neuropathy. $J$ Diabetes Complications 2019;33:182-8.

6 Ko S-uk, Stenholm S, Chia CW, et al. Gait pattern alterations in older adults associated with type 2 diabetes in the absence of peripheral neuropathy--results from the Baltimore Longitudinal Study of Aging. Gait Posture 2011;34:548-52.

7 Crews RT, Yalla SV, Fleischer AE, et al. A growing troubling triad: diabetes, aging, and falls. J Aging Res 2013;2013:1-6.

8 Kirkwood RN, Borém IL, Sampaio RF, et al. Frailty status and gait parameters of older women with type 2 diabetes. Can J Diabetes 2019;43:121-7

9 Wrobel JS, Najafi B. Diabetic foot biomechanics and gait dysfunction. J Diabetes Sci Technol 2010;4:833-45.

10 Prakash C, Kumar R, Mittal N. Recent developments in human gait research: parameters, approaches, applications, machine learning techniques, datasets and challenges. Artif Intell Rev 2018;49:1-40.

11 Mundt M, Thomsen W, Witter T, et al. Prediction of lower limb joint angles and moments during gait using artificial neural networks. Med Biol Eng Comput 2020;58:211-25.

12 Muro-de-la-Herran A, Garcia-Zapirain B, Mendez-Zorrilla A. Gait analysis methods: an overview of wearable and nonwearable systems, highlighting clinical applications. Sensors 2014;14:3362-94.

13 Gor-García-Fogeda MD, Cano de la Cuerda R, Carratalá Tejada M, et al. Observational gait assessments in people with neurological disorders: a systematic review. Arch Phys Med Rehabil 2016;97:131-40.

14 Roberts M, Mongeon D, Prince F. Biomechanical parameters for gait analysis: a systematic review of healthy human gait. Phys Ther Rehabil 2017;4:6.

15 Begg R, Kamruzzaman J. A machine learning approach for automated recognition of movement patterns using basic, kinetic and kinematic gait data. $J$ Biomech 2005;38:401-8.

16 Mannini A, Trojaniello D, Cereatti A, et al. A Machine Learning Framework for Gait Classification Using Inertial Sensors: Application to Elderly, Post-Stroke and Huntington's Disease Patients. Sensors 2016;16:134.

17 König N, Singh NB, Baumann CR, et al. Can gait signatures provide quantitative measures for Aiding clinical decision-making? A systematic meta-analysis of gait variability behavior in patients with Parkinson's disease. Front Hum Neurosci 2016;10:319.

18 Caldas R, Mundt M, Potthast W, et al. A systematic review of gait analysis methods based on inertial sensors and adaptive algorithms. Gait Posture 2017;57:204-10.

19 Moons KGM, Altman DG, Reitsma JB, et al. Transparent reporting of a multivariable prediction model for individual prognosis or diagnosis (TRIPOD): explanation and elaboration. Ann Intern Med 2015;162:W1-73.

20 Wolff RF, Moons KGM, Riley RD, et al. PROBAST: a tool to assess the risk of bias and applicability of prediction model studies. Ann Intern Med 2019;170:51.

21 Khera P, Kumar N. Role of machine learning in gait analysis: a review. J Med Eng Technol 2020;44:441-67.

22 Figueiredo J, Santos CP, Moreno JC. Automatic recognition of gait patterns in human motor disorders using machine learning: a review. Med Eng Phys 2018;53:1-12.

23 Moher D, Shamseer L, Clarke M, et al. Preferred reporting items for systematic review and meta-analysis protocols (PRISMA-P) 2015 statement. Syst Rev 2015;4:1.

24 Rethlefsen ML, Kirtley S, Waffenschmidt S, et al. PRISMA-S: an extension to the PRISMA statement for reporting literature searches in systematic reviews. Syst Rev 2021;10:39.

25 Ouzzani M, Hammady H, Fedorowicz Z, et al. Rayyan-a web and mobile APP for systematic reviews. Syst Rev 2016;5:210.

26 Moons KGM, de Groot JAH, Bouwmeester W, et al. Critical appraisal and data extraction for systematic reviews of prediction modelling studies: the charms checklist. PLoS Med 2014;11:e1001744.

27 Collins GS, Reitsma JB, Altman DG, et al. Transparent reporting of a multivariable prediction model for individual prognosis or diagnosis (TRIPOD): the TRIPOD statement. Ann Intern Med 2015;162:55-63. 